\title{
Voraussetzungen in Perspektivklärungsgruppen - damit ein kindgerechter Aufenthalt gelingt
}

\author{
Sascha Dalügge $\cdot$ Jenni Walther $\cdot$ Johanna Schratz $・$ Nicola Großheinrich
}

Eingegangen: 3. September 2021 / Angenommen: 28. Oktober 2021 / Online publiziert: 9. November 2021

(C) Der/die Autor(en) 2021

Zusammenfassung Perspektivklärungsgruppen der stationären Kinder- und Jugendhilfe müssen sowohl eine tragfähige Einschätzung für den weiteren Lebensweg der Kinder und Jugendlichen entwickeln, Krisen bewältigen als auch zum Gelingen der kindlichen Entwicklung beitragen. In dieser Studie wurden Einschätzungen zu gegenwärtigen Voraussetzungen für die Arbeit in diesen Gruppen, den Prozess der Perspektivklärung und der Lebenssituation der Kinder und Jugendlichen aus Sicht der Mitarbeiter*innen gewonnen. Es zeigte sich Nachbesserungsbedarf im Umgang mit psychisch und sexuell auffällig geltendem Verhalten der Kinder und in der Vernetzung der am Hilfesystem Beteiligten. Zudem wurde offensichtlich, dass Kinder und Jugendliche am Prozess der Perspektivklärung nicht ausreichend beteiligt sind. Veränderungen im Sinne der Kinder und Jugendlichen, aber auch der Fachkräfte sollten angestrebt werden.

Schlüsselwörter Entwicklungsstörungen · Inobhutnahme $\cdot$ Kinder- und Jugendhilfe $\cdot$ Sexualpädagogik · Psychische Störungen · Partizipation

Die Autoren S. Dalügge und J. Walther teilen sich die Erstautor*innenschaft.

Sascha Dalügge, Soziale Arbeit M. A.

Wuppertal, Deutschland

Jenni Walther, Soziale Arbeit M. A.

Münster, Deutschland

Johanna Schratz, Soziale Arbeit M. A./Sozialpädagogik M. A.

Köln, Deutschland

Prof. Dr. Nicola Großheinrich $(\square)$

Abteilung Köln, Katholische Hochschule NRW, Institut für Gesundheitsforschung und Soziale

Psychiatrie (igsp), Köln, Deutschland

E-Mail: n.grossheinrich@katho-nrw.de 


\title{
Conditions in residential groups aimed at clarifying perspectives-ensuring child-supportive stays are successful
}

\begin{abstract}
Clarification of perspectives in youth residential groups must include a reliable assessment for the future life of children and adolescents. Such perspective groups must also manage crises and contribute to the successful development of the children. The present study records the staff's assessments of the current requirements for the work of such groups, the process of clarifying perspectives, and the children's and adolescents' life situation. The results imply that the handling of psychologically and sexually problematic behavior needs to be optimized. Further steps are needed to improve the networking of those involved in the care system. In addition, it became apparent that children and adolescents are not sufficiently involved in clarifying their own perspectives. Efforts should be made to implement improvements for both youth and professionals.
\end{abstract}

Keywords Developmental disorders · Taking into care $\cdot$ Youth welfare $\cdot$ Sex education $\cdot$ Mental disorders $\cdot$ Participation

\section{Hintergrund}

Kinder und Jugendliche, für die angesichts einer Gefährdungssituation oder Problemlage nicht feststeht, ob sie in ihrem bisherigen Umfeld ihre Entwicklung fortsetzen können, werden in Perspektivklärungsgruppen ${ }^{1}$ betreut, unterstützt und gefördert. Den Gruppen kommt dabei nicht nur der Auftrag zu, gemeinsam mit dem Kind oder dem*der Jugendlichen eine tragfähige Perspektive zu erarbeiten und die aktuelle Problemsituation zu entschärfen, sondern entsprechend der Entwicklungsbedarfe und Ressourcen die jungen Menschen zu unterstützen. Wissenschaftlich wurden diese Gruppen, die teils als Intensiv-, teils als Regelwohngruppe konzipiert sind, bislang nur vereinzelt berücksichtigt. Dabei ist der Grundsatz, jedes Kind in seiner Entwicklung zu fördern, bereits im Sozialgesetzbuch festgehalten (§ 1 SGB VIII). Besondere Beachtung ist dem Thema zuzuschreiben, da - wie in der „Mannheimer Risikokinderstudie“ (Esser und Schmidt 2017) festgestellt - ein Heimaufenthalt ein Risikofaktor für eine gelingende Entwicklung ist.

In einer bundesweit durchgeführten Studie zur Gefährdungs- und Perspektivklärung wurden 340 Heimeinrichtungen in eine Untersuchung eingeschlossen (Zitelmann 2013, 2010). Ergebnisse der Studie benennen die personelle Situation sowie die zeitlichen Ressourcen als mangelhaft. Auch die Kompetenzen der Fachkräfte wurden bezüglich der Gefährdungseinschätzung, der therapeutischen und erzieherischen Hilfen als unzureichend beschrieben. Neuere Studien mit einer ähnlichen Schwerpunktsetzung existieren bislang nicht.

\footnotetext{
1 Neben dem Terminus der Perspektivklärungsgruppe bestehen für die in ihrer Aufgabe übereinstimmenden Gruppen Begrifflichkeiten wie Diagnose- oder Clearinggruppe. Auch Inobhutnahmegruppen, die über die Funktion einer Schutzstelle hinausgehen, können zur Perspektivklärung angedacht sein.
} 
Die Bedingungen einer Inobhutnahme können sich aufgrund der hohen Fluktuation der Kinder, der altersgemischten Gruppen und der wechselnden Fachkräfte im Schichtdienst belastend auf die Kinder und Jugendlichen auswirken (Petermann et al. 2014). Dennoch wird nahegelegt, dass ein Heimaufenthalt im Falle einer kurzfristigen Unterbringung die ,am wenigsten schädliche Alternative“ (Schleiffer 2018, S. 136) sei (auch Neumann-Witt 2020), auch wenn empirische Belege fehlen. Weiterhin zeigen Untersuchungen, dass Kinder und Jugendliche ihrer Wahrnehmung nach in über der Hälfte der Fälle (52\%) nicht in wichtige sie betreffende Entscheidungen einbezogen worden sind (Rücker et al. 2015a).

Zur pädagogischen Begleitung der Kinder und Jugendlichen sind laut Petermann et al. (2014) adäquate Konzepte sowie ein gut ausgebildetes Fachpersonal notwendig, um angemessen auf die psychischen Belastungen der aufgenommenen jungen Menschen reagieren zu können. In einer Untersuchung wiesen über $70 \%$ der Kinder und Jugendlichen klinisch auffällige Ergebnisse auf (Schmid 2012). Auch in Studien aus Spanien (Martín et al. 2019) und Norwegen (Jozefiak et al. 2016) wird auf die erhöhte Prävalenz psychischer Störungen in der stationären Kinder- und Jugendhilfe hingewiesen. Zugleich kann jedoch festgehalten werden, dass Kinder und Jugendliche mit stark psychisch abweichendem Verhalten besonders von Maßnahmen der Kinder- und Jugendhilfe profitieren (Fegert und Petermann 2014). In einer im Bundesland Bayern durchgeführten Studie (Beck 2015) wurde darüber hinaus gezeigt, dass Kinder und Jugendliche vor oder nach einem psychiatrischen Aufenthalt oftmals eine Jugendhilfemaßnahme wahrgenommen haben. Damit deutet sich an, dass für die betreffenden Berufsgruppen aus Psychiatrie und Kinder- und Jugendhilfe neben Fortbildungen für das Personal auch Kooperationen zwischen den Einrichtungen angemessen sein könnten. Mit einem kooperativen Behandlungsmodell im Rahmen des Ulmer Heimkinderprojekts, welches sich auf gemeinsame Fallbesprechungen, interdisziplinäre Therapieziele, Versorgung wie Evaluation beruft und ergänzend psychiatrisch-psychotherapeutische Interventionen bereithält, konnte nachgewiesen werden, dass sich auf diese Weise die Dauer der psychiatrischen Aufenthalte verringern lässt (Goldbeck et al. 2009). Allerdings zeigen Forschungsergebnisse, dass lediglich die Hälfte der Befragten die Kooperation zwischen den Fachrichtungen Sozialpädagogik und Psychiatrie/Psychotherapie auf Augenhöhe erleben (MüllerLuzi und Schmid 2017). Damit Kooperation als Problemlösungsstrategie wirken könne, seien zeitliche Ressourcen und eine inhaltliche, fallorientierte Verständigung der Kooperationspartner*innen notwendig (Loch 2018). Ähnlich verhält es sich mit einer finnischen Studie (Timonen-Kallio 2019) zur Zusammenarbeit zwischen dem Gesundheitssystem und der stationären Kinder- und Jugendhilfe, die für die Erweiterung gleichberechtigter interprofessioneller Kooperation wirbt. Als Stolpersteine in der Kooperation können entsprechend einer Projektanalyse (Fischer und Geene 2019) ein uneinheitliches Kooperationsverständnis, eine geringe Nachhaltigkeit und fehlende Ressourcen betrachtet werden. Ein Gelingen hänge oft von den Rahmenbedingungen, dem vorherrschenden Engagement sowie Kompetenz in der Netzwerkkoordination ab.

Der Arbeit mit den Erziehungsberechtigten ist eine hohe Bedeutung zuzusprechen, auch vor dem Hintergrund, dass 2017 etwa $43 \%$ der Kinder und Jugendlichen in die Herkunftsfamilie zurückkehrten (Statistisches Bundesamt 2018). Ein gelingen- 
der Einbezug der Erziehungsberechtigten kann sich derweil zum einen auf die Dauer und zum anderen auf das planmäßige Abschließen der Hilfemaßnahme auswirken (Petermann et al. 2014; Institut für Kinder- und Jugendhilfe [IKJ] 2015). Dafür werden von Seiten der Erziehungsberechtigten Kommunikation, Transparenz, Involviertheit sowie die Akzeptanz, als Prozessbeteiligte wahrgenommen zu werden, als notwendig angesehen (Pavel 2016). Grundsätzlich, so die Schlussfolgerungen einer qualitativen Interviewstudie (Mayer 2017), weist dieser Bereich einen hohen Innovations- und Veränderungsbedarf auf. Hierbei müsse der Kooperationsbereitschaft aller Beteiligten einem professionellen Selbstverständnis der Fachkräfte sowie einer in den Einrichtungen konzeptionell verankerten Transparenz eine hohe Bedeutung für die Zusammenarbeit zugeschrieben werden. Zugleich wird, wie eine Studie von Kaufhold et al. (2016) zeigt, der Durchführung von Umgangskontakten (94\%) von befragten Fachkräften ein hoher Stellenwert attestiert.

Nach einer Studie von Rudeck et al. (2008) schätzen lediglich $38 \%$ der im stationären Bereich untergebrachten Kinder und Jugendlichen die Beteiligungsmöglichkeit als gut ein. Im Alltagsgeschehen würden monetäre Einsparungen, der Zeitdruck und die Anforderung, einen unmittelbaren Wirkungsnachweis zu erbringen, die Beteiligung der Kinder erschweren (Gadow et al. 2013). Dabei benötigt es eine den Kindern und Jugendlichen zugesprochene Expert*innenposition in der Hilfeplanung, um Teilhabe zu erreichen (Sinclair et al. 2019).

Kindliche Sexualität wird von der empirischen Sozialforschung bislang wenig beachtet, sofern der Untersuchungsgegenstand nicht primär sexualisiertes Verhalten oder sexueller Missbrauch an und von Kindern ist (Schuhrke 2015; Bundschuh 2010). Hintergrund könnte das erhöhte Risiko für sexuell grenzverletzende Erfahrungen fremduntergebrachter Kinder und Jugendlicher sein (u. a. Helming et al. 2011 oder Bundschuh 2010, im nichtdeutschsprachigen Raum u. a. McKibbin (2017) oder Euser et al. (2013)). In einer Studie von Helming et al. (2011) wiesen 39\% der Heimeinrichtungen in den vorangegangenen drei Jahren mindestens einen Verdachtsfall von sexueller Gewalt unter Gleichaltrigen auf. Im Rahmen einer Dunkelfelderhebung gaben $57 \%$ der befragten Bewohner*innen an, sexuelle Gewalt vor oder während ihres Aufenthaltes erlebt zu haben (Allroggen et al. 2016). Durch die Auswertung von Daten aus dem EVAS-Verfahren (Evaluationssystem erzieherischer Hilfen) lässt sich erkennen, dass $14,9 \%$ der Kinder und Jugendlichen die Hilfen nach $§ 34$ SGB VIII erhalten, ein sexuell auffällig geltendes Verhalten aufweisen (Schuhrke und Arnold 2009). Die Gruppe dieser Kinder wird als belasteter beschrieben als Kinder und Jugendliche mit anderen Auffälligkeiten (ebd.; auch Jud et al. 2016).

Vor diesem Hintergrund erscheint es umso bedeutender, eine gelingende $\mathrm{Be}$ gleitung kindlicher Sexualentwicklung und das Schaffen eines sexualfreundlichen Klimas zu thematisieren. Eine qualitative Studie aus Belgien, Dänemark und den Niederlanden (Walpot et al. 2017) konnte erste Hinweise benennen, welche Kompetenzen Fachkräfte in der Arbeit mit fremduntergebrachten Kindern und Jugendlichen benötigen, um diesen eine für den weiteren Lebensverlauf günstige sexuelle Entwicklung zu ermöglichen. Neben einer entsprechenden fachlichen Expertise und einer handlungsbezogenen Integration fachlicher Standards müssten durch den strukturellen Rahmen notwendige Voraussetzungen geschaffen werden. Den zu unterstützenden jungen Menschen solle ein sicheres Umfeld geboten werden, das 
ihnen den Raum für positiv bewertete sexuelle Erfahrungen bietet und ihnen die Möglichkeiten gibt, eigene Normen und Werte auszubilden. Im Rahmen von sexueller Bildung könnten die jungen Menschen nicht nur ihr Wissen ausbauen und Fertigkeiten stärken, sondern auch das Selbstbewusstsein und die Fähigkeit entwickeln, verantwortungsbewusste Entscheidungen zu treffen. Dies wird untermauert durch die „Looking after Children Initiative“, die mehr als 1000 Kinder nach ihren Sichtweisen zu einer gelingenden Heimerziehung befragt (Ofsted 2007, 2010, 2011). Anhand der Ergebnisse wird deutlich, dass der kindlichen Beteiligung, der Informationsweitergabe, den Beschwerdemöglichkeiten und der Achtung der Privatsphäre ein besonders hoher Stellenwert zugeschrieben wird. Weiterhin benannten die Kinder, eine faire Behandlung und Sicherheit in der Einrichtung spüren zu wollen.

Trotz der potenziell enormen Auswirkungen auf den Lebensweg junger Menschen und ihrer Bezugssysteme kann ausgehend von der aktuellen Studienlage die Forschung zu Perspektivklärungsgruppen als nicht ausreichend abgedeckt angesehen werden. Studienergebnisse vermischen sich mit Erkenntnissen zur Heimerziehung und sind wenig spezifisch für Perspektivklärungsgruppen übertragbar. Durch die vorliegende explorative Studie sollen spezifische Einschätzungen durch die Fachkräfte zu den Voraussetzungen in Perspektivklärungsgruppen gewonnen werden, um in Zukunft eine gelingende Arbeit vor dem Hintergrund optimierter kindlicher Entwicklungsbedingungen zu ermöglichen.

\section{Methodik}

Das Ziel der vorliegenden Studie war es, die Bedingungen in Perspektivgruppen aus der Sicht der Mitarbeiter*innen zu erfassen. Besondere Berücksichtigung fand im Rahmen der Studie der Umgang mit psychisch abweichendem Verhalten der Kinder sowie der kindlichen Sexualität. Weiterhin wurden die Vernetzung mit Akteur*innen der Kinder- und Jugendhilfe sowie der Einbezug der Erziehungsberechtigten und schließlich die Partizipationsmöglichkeiten der untergebrachten Kinder untersucht. Zielgruppe dieser Studie waren Fachkräfte, die in einer Gruppe arbeiteten, in welcher eine am Bedarf des Kindes orientierte Zukunftsperspektive entwickelt werden sollte. Zunächst wurden die Fachkräfte als Expert*innen interviewt, um in einem zweiten Schritt aus der systematischen Auswertung dieser qualitativen Daten einen Fragebogen für die quantitative Erhebung zu entwickeln (,,sequentiell mixed design“). Eingeschlossen wurden Mitarbeiter*innen von Perspektivgruppen, in denen die Aufenthaltsdauer der betreuten Kinder einen kurz- bis mittelfristigen Zeitrahmen (ein Monat bis zwei Jahre) in der Regel nicht überschritt. Das Konzept der Gruppe sollte sich vorrangig an Kinder zwischen null und 13 Jahren richten. Nicht berücksichtigt wurden aus Gründen der besseren Vergleichbarkeit erstens Clearinggruppen für unbegleitete minderjährige Geflüchtete nach § 42a SGB VIII, zweitens reine Inobhutnahmegruppen ohne einen Klärungsauftrag für eine längerfristige Perspektive und drittens Wohngruppen der stationären Kinder- und Jugendhilfe, in denen nur vereinzelt Plätze zur Perspektivklärung angeboten wurden.

Zur Erfassung möglichst aller in Nordrhein-Westfalen ansässigen Perspektivklärungsgruppen wurden die Jugendämter in NRW um eine Auflistung der innerhalb 
ihrer jeweiligen örtlichen Zuständigkeit liegenden Perspektivklärungsgruppen gebeten. Weiterhin wurde das aktuelle Rheinische Jugendhilfeverzeichnis und eine vom Landschaftsverband Westfalen-Lippe (LWL) zur Verfügung gestellte Liste mit Einrichtungen der stationären Kinder- und Jugendhilfe nach Perspektivklärungsgruppen überprüft und entsprechend gefiltert. Durch diesen Prozess wurden 82 stationäre Kinder- und Jugendhilfeeinrichtungen ermittelt, die insgesamt 124 den Kriterien entsprechenden Perspektivklärungsgruppen aufwiesen. Nach Ablauf des Befragungszeitraums lagen 84 vollständige und den Kriterien entsprechende Fragebögen vor.

Für die quantitative Befragung wurden die Bereichsleiter*innen der jeweiligen Gruppe im Vorfeld informiert und erhielten einen Onlinelink zu einem anonymisierten Fragebogen (LimeSurvey Version 2.73.1+171220), der allen Mitarbeiter*innen der Perspektivklärungsgruppen in NRW die Möglichkeit geben sollte, an der Befragung teilzunehmen. Die subjektive Sichtweise der Fachkräfte wurde mittels 5-stufiger Likert-Skalen erfragt ( $1=$ vollkommene Zustimmung bis $5=$ überhaupt nicht). Weiterhin wurden freie Eingabefelder, Rangordnungen und Fragen mit Mehrfachantwortmöglichkeiten genutzt.

\section{Ergebnisse}

\subsection{Psychisch abweichendes Verhalten als Aspekt der Perspektivklärungsgruppe}

Die Mitarbeiter*innen wurden gebeten, anzugeben, mit welchen klinisch-psychiatrischen Störungsbildern sie in der Regel in Kontakt kommen und wie sicher sie sich im Umgang mit diesen fühlen (Tab. 1).

Zugleich nannten befragte Fachkräfte, dass sie besonders Weiterbildungen im Bereich der Traumapädagogik (96,4\%), der Sexualpädagogik (92,9\%), der Deeskalation $(91,7 \%)$ und der Systemischen Beratung $(86,9 \%)$ für sinnvoll halten.

Bestehende Diagnosen nach dem ICD-10 wurden von 45,2\% der Teilnehmer*innen im Team kritisch hinterfragt. 61,9\% ließen ggf. Diagnosen aktualisieren, die durch Vorinformationen vorlagen. Falls das Kind bei der Aufnahme in die Gruppe Medikamente einnahm, wurde von zwei Dritteln $(65,5 \%)$ der Fachkräfte die Notwendigkeit einer Neuvorstellung diskutiert. $44 \%$ waren mit den (Neben-)Wirkungen der Medikamente vertraut.

\subsection{Kindliche Sexualität und sexualisiertes Verhalten als Aspekt der Perspektivklärungsgruppe}

Sechs von zehn Fachkräften (58,3\%) gaben an, dass sexualpädagogische Themen konzeptionell verankert sind. Das Themenfeld „Sexuelle Bildung“ war nach Einschätzung der Hälfte der Fachkräfte (50\%) und das Themenfeld „Sexualerziehung“ bei $56 \%$ des pädagogischen Personals Bestandteil der Arbeit.

Bei der Befragung zu spezifischen sexualpädagogischen Themen schätzten die Befragten ein, inwiefern sich diese in ihrem Arbeitsalltag wiederfinden lassen und wie sicher sie sich damit fühlen (Tab. 2). 


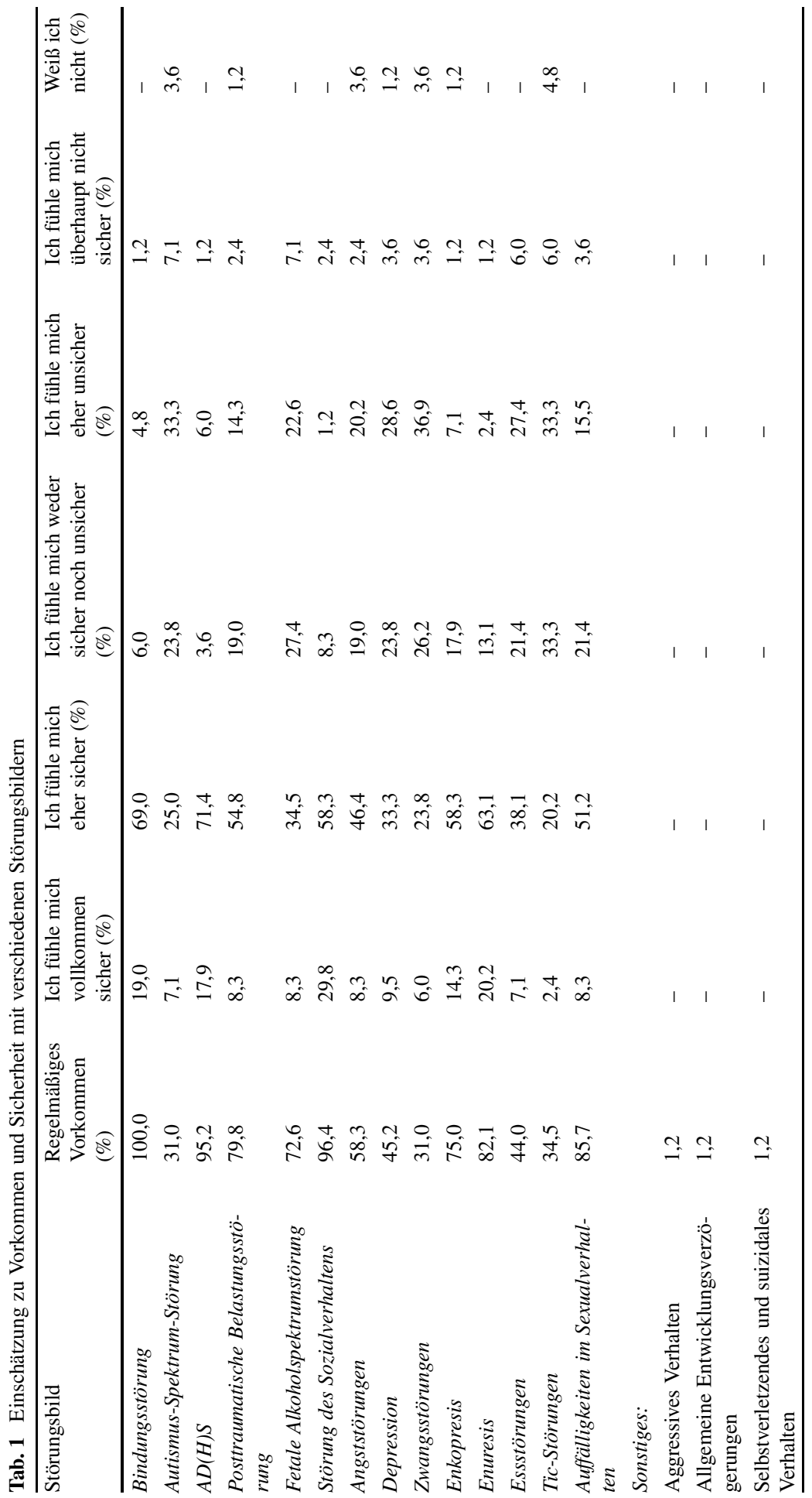




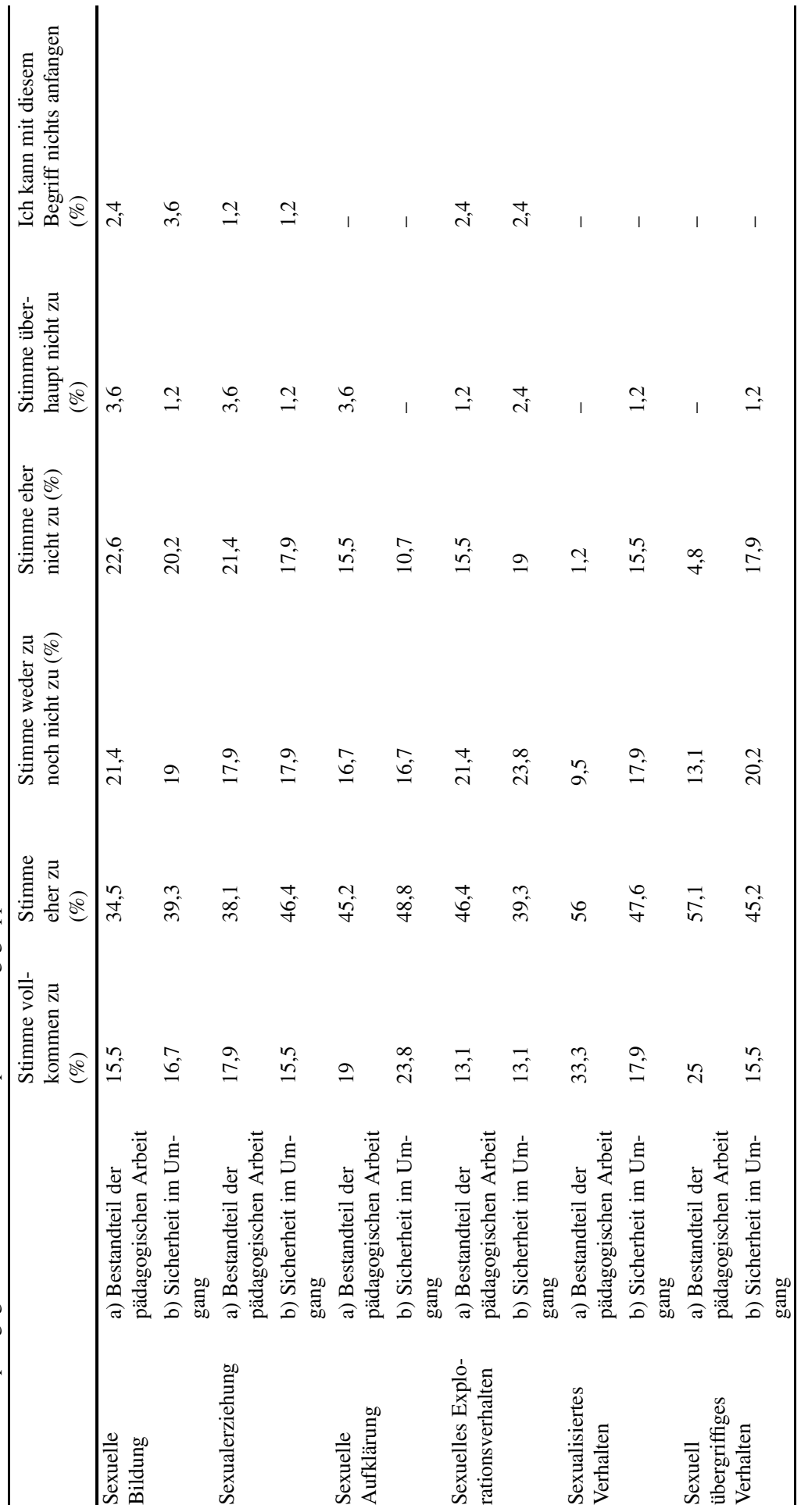


Insgesamt 92,9\% aller Studienteilnehmer*innen erachteten eine sexualpädagogische Weiterbildung für ihre Gruppe als sinnvoll.

\subsection{Vernetzung mit Akteur*innen der Kinder- und Jugendhilfe}

Für die Perspektivklärungsgruppe erachteten annähernd alle Befragten (92,9\%) ein interdisziplinäres Team als notwendig. Wie die Vernetzung zu Kooperationspartner*innen aus den unterschiedlichsten Professionen außerhalb der eigenen Einrichtung bewertet wurde, stellt einen weiteren Schwerpunkt der Forschung dar. Die Vernetzung zum Jugendamt wurde von drei Vierteln der Befragten (76,2\%) als gut beschrieben. Schwierigkeiten in der Kooperation benannten 36,9\%. Eine detaillierte Übersicht zur Bewertung einzelner Bereiche lässt sich Tab. 3 entnehmen. Zwei Drittel der Befragten (69\%) sind der Meinung, die Empfehlung über die Perspektive des Kindes, die im Rahmen der pädagogischen Diagnostik entwickelt wird, werde beim Jugendamt ernst genommen.

Einen Bereich, der in der Vernetzung von vier von fünf Fachkräften $(79,8 \%)$ als gut bewertet wurde, stellte die Schule dar. Als überwiegend gut wurde die Vernetzung zu Ärzt*innen beschrieben (82,1\%). Die Zusammenarbeit mit Fachpersonal zur psychologischen Diagnostik wurde von zwei Dritteln $(63,1 \%)$ und die Vernetzung zur Kinder- und Jugendpsychiatrie von der Hälfte der Befragten (50\%) als gut bewertet.

\subsection{Zusammenarbeit mit den Erziehungsberechtigten}

Für eine erfolgreiche Perspektivklärung mussten nach Einschätzung von neun von zehn Fachkräften $(90,5 \%)$ die Erziehungsberechtigten einbezogen werden. In der Regel $(86,9 \%)$ fand diese Arbeit auch dann statt, wenn keine Rückführung des Kindes in seine Herkunftsfamilie angedacht ist. Etwa die Hälfte $(54,8 \%)$ war mit der Form der Zusammenarbeit zufrieden.

Die Teilnehmer*innen wurden gebeten, Fragen zur Zusammenarbeit mit den Erziehungsberechtigten durch Mehrfachantwortmöglichkeiten anzugeben: Bei der Mehrzahl der Befragten (88,1\%) fand die Arbeit mit den Erziehungsberechtigten

Tab. 3 Schwierigkeiten in der Zusammenarbeit mit dem Jugendamt

\begin{tabular}{|c|c|c|c|c|c|c|}
\hline $\begin{array}{l}\text { Bereiche der Zusam- } \\
\text { menarbeit }\end{array}$ & $\begin{array}{l}\text { Stimme } \\
\text { vollkom- } \\
\text { men zu } \\
(\%)\end{array}$ & $\begin{array}{l}\text { Stimme } \\
\text { eher zu } \\
(\%)\end{array}$ & $\begin{array}{l}\text { Stimme } \\
\text { weder zu } \\
\text { noch nicht } \\
\text { zu (\%) }\end{array}$ & $\begin{array}{l}\text { Stimme } \\
\text { eher } \\
\text { nicht zu } \\
(\%)\end{array}$ & $\begin{array}{l}\text { Stimme } \\
\text { überhaupt } \\
\text { nicht zu } \\
(\%)\end{array}$ & $\begin{array}{l}\text { Weiß } \\
\text { ich } \\
\text { nicht } \\
(\%)\end{array}$ \\
\hline Informationsfluss & 9,5 & 48,8 & 13,1 & 27,4 & 1,2 & - \\
\hline Kooperationsbereitschaft & 7,1 & 29,8 & 17,9 & 36,9 & 8,3 & - \\
\hline $\begin{array}{l}\text { Transparenz bzgl. der } \\
\text { Perspektiventscheidung }\end{array}$ & 7,1 & 36,9 & 19 & 29,8 & 7,1 & - \\
\hline Erreichbarkeit & 20,2 & 44 & 19 & 11,9 & 4,8 & - \\
\hline $\begin{array}{l}\text { Klare Auftragsformu- } \\
\text { lierung }\end{array}$ & 14,3 & 31 & 22,6 & 25 & 7,1 & - \\
\hline $\begin{array}{l}\text { Beantragung von Gel- } \\
\text { dern }\end{array}$ & 10,7 & 35,7 & 19 & 23,8 & 8,3 & 2,4 \\
\hline
\end{tabular}


zum Erhebungszeitraum nach Bedarf und bei der Hälfte $(51,2 \%)$ bei Gelegenheit statt. Drei von vier Mitarbeiter*innen (75\%) gaben an, die Zusammenarbeit mit den Erziehungsberechtigten werde durch telefonische Termine abgedeckt. $81 \%$ sagten aus, die Arbeit mit den Erziehungsberechtigten fände vor und 77,4\% nach den Besuchskontakten statt. $44 \%$ gaben an, die Arbeit mit den Erziehungsberechtigten sei durch regelmäßige Einzeltermine geregelt.

$\mathrm{Zu}$ den Inhalten der Zusammenarbeit zählten das Herausarbeiten der Ressourcen der Kinder $(85,7 \%)$ und die Auseinandersetzung mit dem Beziehungsverhalten zwischen Erziehungsberechtigten und Kind $(85,7 \%)$. Vier von fünf Mitarbeiter*innen $(79,8 \%)$ thematisierten den aktuellen Entwicklungsstand des Kindes, drei Viertel $(75 \%)$ klärten die Eltern über kindliche Verhaltensweisen/Störungsbilder (Psychoedukation) auf. Netzwerkaufbau/-stärkung $(38,1 \%)$ sowie eine Veränderung des häuslichen Umfeldes (51,2\%) wurde nach deskriptiver Auswertung weniger häufig in der Arbeit mit den Erziehungsberechtigten angegeben. 3,6\% gaben an, die Arbeit mit den Erziehungsberechtigten sei kein Bestandteil ihres Tätigkeitsfeldes. $56 \%$ der Fachkräfte schätzten die Kooperationsbereitschaft der Erziehungsberechtigten als schwierig ein. Die Beteiligung der Erziehungsberechtigten (45,2\%) sowie die Neutralität gegenüber dem Familiensystem wurde von Seiten der Mitarbeiter*innen als schwierig beschrieben $(36,9 \%)$.

Nahezu alle befragten Fachkräfte $(95,2 \%)$ erachteten es in der Zusammenarbeit mit den Erziehungsberechtigten als erstrebenswert, Transparenz bezüglich der Perspektivklärung des Kindes herzustellen. Sieben von zehn Teilnehmer*innen (72,6\%) waren der Meinung, die Erziehungsberechtigten sollten die Kinder im Beisein von Mitarbeiter*innen der Gruppe zu Terminen (z. B. Arztbesuche) begleiten. Mehr als zwei Drittel (69,0\%) erachteten es als sinnvoll, dass während der Besuchskontakte eine Anleitung durch Fachkräfte der Gruppe stattfände; ein Viertel (25\%), dass eine Trainingswohnung an die Perspektivklärungsgruppe angegliedert wird. Etwas mehr als die Hälfte der Befragten (54,8\%) plädierte dafür, Gespräche mit den Erziehungsberechtigten in deren Haushalt abzuhalten.

\subsection{Partizipationsmöglichkeiten in der Perspektivklärungsgruppe}

Im Zuge einer möglichen Aufnahme in die Perspektivklärungsgruppe konnte etwa jede fünfte Fachkraft $(22,6 \%)$ die Sichtweise des Kindes berücksichtigen. Eine altersentsprechende Partizipation während des Aufenthaltes war nach Auffassung der Mitarbeiter*innen hinsichtlich individueller Absprachen (92,9\%), Einzelaktivitäten $(88,1 \%)$ und Gruppenaktivitäten $(85,7 \%)$ möglich. Weiterhin konnten die Kinder an der Zimmergestaltung (78,6\%), dem Tagesablauf (50\%), der Gruppenausstattung $(41,7 \%)$ sowie den Gruppenregeln $(31 \%)$ mitwirken. Bezüglich der Perspektivempfehlung des Kindes gaben $44 \%$ und betreffend der Perspektiventscheidung ein Viertel der Fachkräfte (25\%) an, dass die Kinder hieran teilhaben könnten.

Die Teilnehmer*innen der Studie wurden gebeten, eine Rangordnung zu erstellen, wodurch sich ihre Arbeit begründen lasse: Ein Drittel (33,3\%) der Befragten wählte den Auftrag des Jugendamtes auf Rang 1 oder 2. Voranging (Rang 1 und 2) formulierte sich für $23,8 \%$ der Fachkräfte ihre Arbeitsgrundlage aus der eigenen professionellen Position heraus, etwa eine*r von vier Teilnehmer*innen $(26,1 \%)$ 
gab an, diese ergebe sich aus den Vorgaben der Einrichtung. Lediglich eine*r von sieben $(16,7 \%)$ sah den Wunsch des Kindes auf Rang 1 oder 2.

\section{Diskussion}

In der Gesamtbetrachtung weisen die Ergebnisse auf einen strukturellen Handlungsbedarf hin bezogen auf die Perspektivklärung sowie deren Vernetzung (36,9\% der Fachkräfte beschrieben generelle Schwierigkeiten in der Kooperation zum Jugendamt) und die Zusammenarbeit mit den Erziehungsberechtigten (lediglich 54,8\% ist mit der gegenwärtigen Form der Zusammenarbeit zufrieden). Darüber hinaus zeigt sich, dass Kinder oftmals an der Entwicklung (44\%) und Verwirklichung (25\%) ihrer Perspektive äußerst begrenzt teilhaben können. Auch fehlt es Fachkräften an Sicherheit, um mit sexuell sowie psychisch auffällig geltendem Verhalten umzugehen. So wünschten sich 96,4\% Weiterbildungen im Bereich der Traumapädagogik und $92,9 \%$ im Bereich der Sexualpädagogik.

Kindern wird vom Gesetz das Recht zugesprochen, in ihrer Entwicklung gefördert zu werden. Zusätzlich hat die Kinder- und Jugendhilfe den Auftrag, Benachteiligungen abzubauen ( $\$ 1$ SGB VIII). Die vorgestellten Studienergebnisse können daher als Handlungsauftrag verstanden werden, damit erstens Berufsanfänger*innen während ihres Studiums oder ihrer Ausbildung zielgerichtet vorbereitet werden, zweitens Einrichtungen auf die Bedarfe ihrer Mitarbeiter*innen hinsichtlich struktureller Merkmale und fachlicher Qualifikation wirken und drittens Vorgaben durch die Politik gesetzlich festgehalten und dazugehörige Ressourcen bereitgestellt werden.

\subsection{Umgang mit psychisch abweichendem Verhalten der Kinder}

Bei der Betrachtung von psychisch abweichenden Verhaltensweisen in Perspektivklärungsgruppen fällt nicht nur auf, dass viele Fachkräfte das Auftreten dieser kindlichen Verhaltensweisen beschreiben, sondern auch, dass sie sich oft nicht in gleichem Maße im Umgang mit diesen sicher fühlen. Eine uneinheitliche und nicht ausreichende Qualifikation wurde bereits durch Zitelmann (2013) bemängelt. In diesem Zusammenhang ist darauf hinzuweisen, dass ein Nachbesserungsbedarf im Rahmen des Studiums oder der Ausbildung wünschenswert wäre. Bedeutend wird dieses Thema, wenn psychische Auffälligkeiten, Traumabelastungen, Suizidalität oder selbst- sowie fremdgefährdende Verhaltensweisen der Kinder übersehen werden und dadurch eine geeignete Versorgung nicht gewährleistet wird (Rücker et al. 2015b).

Auch der kritische Umgang mit Diagnosen könnte in diesem Rahmen thematisiert werden, damit defizitorientierte Zuschreibungen weder in einem negativen Selbstbild der Kinder noch einseitigen Betrachtungen durch das Umfeld münden. Folglich würden kindliche Verhaltensweisen nicht als feststehende Determinanten gesehen werden. Es gilt, dass auf der einen Seite symptomatische Verhaltensweisen einbezogen werden müssen, auf der anderen Seite stellen sie nur einen Teil des Kindes dar (Baierl 2014). 
Inwiefern durch Kooperationen zur Kinder- und Jugendpsychiatrie oder einer psychotherapeutischen Anbindung eine Krisenbewältigung während des zeitlich befristeten Aufenthalts in der Perspektivklärungsgruppe unterstützt werden kann oder ob Krisen und Belastungen während dieser Zeit effektiver durch die sozialtherapeutischen Kompetenzen der Fachkräfte in der Gruppe aufgefangen werden können, müssen vertiefende Untersuchungen zeigen.

\subsection{Achtung kindlicher Sexualität}

Die Bedeutung von Sexualität in der stationären Kinder- und Jugendhilfe wird von Fachkräften hinsichtlich problematisch erlebten Sexualverhaltens hoch eingeschätzt. Es könnte beanstandet werden, dass lediglich 58,3\% der Mitarbeiter*innen vom sexualpädagogischen Konzept ihrer Einrichtung wissen, obwohl Sexualität von frühster Kindheit an eine hohe Bedeutung zugesprochen wird (WHO und BZgA 2011) und gemäß den Vorgaben des Landesjugendamtes jede Einrichtung verpflichtet ist, konzeptionelle Überlegungen zu Sexualpädagogik zu treffen (LWL 2013). Auch ist für nur die Hälfte (50\%) der Mitarbeiter*innen die sexuelle Bildung ein Bestandteil ihrer Arbeit. Diskurse aus der Diskrepanz zwischen formalen Anforderungen und der Umsetzungen in den Gruppen könnten sowohl in den Gruppen als auch in der Ausbildung der Fachkräfte erfolgen. Einen ersten Ansatzpunkt könnte hierbei das Bewusstmachen der identitätsstiftenden, schützenden und ermächtigenden Wirkung von Sexualität für Menschen jeden Alters darstellen.

Zusätzlich ist zu beachten, dass Auffälligkeiten im Sexualverhalten von einem großen Anteil $(85,7 \%)$ der Mitarbeiter*innen beschrieben wurden, sich aber weit weniger im Umgang mit den beschriebenen Verhaltensweisen sicher fühlten $(59,5 \%)$. Deshalb ist es wünschenswert, dem Anliegen der Fachkräfte nach sexualpädagogischen Weiterbildungen $(92,9 \%$ ) nachzukommen sowie die bestehenden Konzepte der Einrichtungen gemeinsam durch Expert*innen und die jeweiligen Fachkräfte der Gruppen zu evaluieren, um diese anschließend verstärkt in den pädagogischen Alltag zu integrieren.

\subsection{Vernetzung als Ressource}

Eine Vernetzung zu kooperierenden Institutionen und der Einbezug einer interdisziplinären Sichtweise stellt ein fundamentales Instrument für ein möglichst umfassendes Bild kindlicher Ressourcen, Potenziale, Belastungsmomente und Herausforderungen in der Perspektivklärung dar. Anhand der Ergebnisse wird besonders ein Handlungsbedarf bezüglich der Zusammenarbeit mit dem Jugendamt sichtbar. Eine naheliegende Konsequenz wäre, eine Häufung der Kontakte zwischen Jugendamt und Einrichtung zu gewährleisten. Durch einen verbesserten zielgerichteten Austausch könnten zugleich die Ressourcen und Erkenntnisse des sozialpädagogischen Fachpersonals gezielt genutzt und die Ergebnisse der sorgfältigen Perspektivklärung einbezogen werden. Vermutlich könnten von dieser Zusammenarbeit nicht nur die Kooperationspartner*innen, sondern auch die Kinder und Jugendlichen profitieren.

Während die Zusammenarbeit mit Schulen wie auch die Vernetzung mit Ärzt*innen eine vorsichtig optimistische Betrachtungsweise zulassen, scheint diese 
Studie - ähnlich wie in vorangegangenen Studien (Müller-Luzi und Schmid 2017; Groen und Jörns-Presentati 2014) - auf einen Verbesserungsbedarf hinsichtlich der Kooperation mit der Kinder- und Jugendpsychiatrie hinzuweisen. Das Eingehen auf und Annehmen der sozialpädagogischen Kompetenzen könnte eine notwendige Ressource für ein sich ergänzendes, fachliches Vorgehen sein (siehe 4.1). Kapazitäten, um weitere Beteiligte einzubeziehen, die dem Hilfeprozess dienlich wären, könnten durch klare arbeitsstrukturierende Maßnahmen geschaffen werden.

\subsection{Einbezug der Erziehungsberechtigten}

Obwohl sowohl in dieser Studie durch das pädagogische Personal als auch in aktuellen Veröffentlichungen (Knuth und Riehm 2020; Pauls 2011) der Zusammenarbeit mit den Erziehungsberechtigten für eine gelingende Perspektivklärung eine hohe Bedeutung zugesprochen wird, bemängeln befragte Fachkräfte zugleich die oft nur unzureichend vorhandenen institutionellen Ressourcen. Vor diesem Hintergrund muss benannt werden, dass Hilfeabbrüche und Misserfolge teils auf eine mangelnde Kooperation zwischen Fachkraft und Familiensystem sowie eine unzulängliche Prozessqualität zurückzuführen sind (Ortmann und Röh 2017).

Feste Termine und die personellen Möglichkeiten, Besuchskontakte zwischen Erziehungsberechtigten und dem Kind regelmäßig durch eine Fachkraft zu begleiten, scheinen naheliegende Ansätze, um einen gelingenden Einbezug der Erziehungsberechtigten zu ermöglichen. Auch die Einrichtung einer Trainingswohnung oder das Einstellen einer Fachkraft, die gezielt die Arbeit mit den Erziehungsberechtigten steuert, könnte zu Fortschritten in diesem Bereich führen.

Auf fachlicher Ebene thematisierten fast die Hälfte der Befragten Schwierigkeiten, die Erziehungsberechtigten partizipieren zu lassen $(45,2 \%)$ und dem Familiensystem gegenüber Neutralität zu wahren $(36,9 \%)$. Über die Hälfte $(56 \%)$ der Fachkräfte berichteten, dass sie die Kooperationsbereitschaft der Erziehungsberechtigten als problematisch erleben. Damit die Arbeit mit den Erziehungsberechtigten von professioneller Seite trotzdem produktiv, transparent und authentisch verlaufen kann, ist eine Sensibilisierung für die Situation der Erziehungsberechtigten in dem Spannungsfeld des Hilfe-Kontroll-Systems naheliegend. Auf fachlicher Ebene könnte thematisiert werden, dass Gespräche mit den Erziehungsberechtigten auf beiden Seiten von Befürchtungen, Vorstellungen und Erwartungen geprägt sind.

\subsection{Achtung von Partizipation}

In den Perspektivklärungsgruppen scheint es in einigen, jedoch nicht in allen Belangen möglich, die Kinder im Alltag am Gruppengeschehen und Entscheidungen partizipieren zu lassen. Allerdings werden dem Kind oftmals weder bei der Aufnahme noch bei der Perspektiventscheidung Einflussmöglichkeiten gegeben. Bereits vorangegangene Studien (Rücker und Büttner 2017; Rücker et al. 2015a; Rudeck et al. 2008) haben auf Defizite in diesem Bereich hingewiesen. Nach Gadow et al. (2013) sei das Alter der Kinder und Jugendlichen ein immer wieder aufkommendes Argument für das Fehlen der Mitbestimmung. Als Ergebnis der vorliegenden Studie sahen die Fachkräfte ihre Arbeitsgrundlage für die Perspektivklärung mehrheitlich 
aus den Vorgaben des Jugendamtes (33,3\%) und der Einrichtung (26,1\%), erst dann aus ihrer fachlichen Position $(23,8 \%)$ heraus und schließlich aus dem Auftrag des Kindes $(16,7 \%)$. Dem entgegen muss auf die hohe Bedeutung von Selbstwirksamkeitserfahrungen für die Identitätsstiftung hingewiesen werden. Weiterhin gilt es zu bedenken, dass sich Partizipation als bedeutender Schutzfaktor gegen sexuelle Gewalt erwiesen hat (Allroggen et al. 2016). Partizipation als Zielsetzung auszurufen, reicht, wie die Resultate der vorliegenden Studie zeigen, nicht aus. Eine Stärkung kindlicher Partizipation beginnt bei der Haltung der Fachkräfte.

\subsubsection{Resümee und Ausblick}

Die Ergebnisse dieser Studie legen dar, dass in dem spezifischen Feld der Perspektivklärungsgruppen Weiterentwicklungen erfolgen sollten, damit Kinder in diesen Gruppen sowohl emotional sicher aufgefangen als auch in ihrer gesamten Entwicklung gefördert werden können. Zu bedenken ist, dass Gruppen, welche die Aufgabe der Perspektivklärung gemein haben, teils enorme Unterschiede und Defizite auf struktureller und fachlicher Ebene aufweisen. Für einen gelingenden Aufenthalt der Kinder wäre es wünschenswert, dass sich strukturelle Begebenheiten unter fachlichen Gesichtspunkten begründen lassen. Spezifisches basis- und handlungsbezogenes Wissen sollte durch alle Mitarbeiter*innen ausreichend verfügbar sein. Damit kann der Diskrepanz zwischen der Prävalenz von und einem sicheren Umgang mit auffällig geltendem Verhalten der Kinder durch eine entsprechende fachliche Qualifizierung entgegengewirkt werden.

Es scheint angezeigt, konkrete Maßnahmen durch Handlungsleitlinien im konzeptionellen, Vernetzungs- sowie Qualifizierungsbereich zu treffen, um generelle Verbesserungen zu erzielen und die bestehenden Zielsetzungen zu erreichen. Konkret bedeutet dies, gemeinsam ein Gruppenklima zu schaffen, welches Partizipation ebenso wie kindliche Sexualität erlaubt und fördert. Zugleich sollte kindliches Verhalten jeglicher Art bestmöglich verstanden werden, damit geeignete sozialpädagogische Interventionen Anwendung finden können. Das Angebot von tätigkeitsspezifischen Weiterbildungen spielt hierbei eine tragende Rolle. Ergänzend könnten Strukturen geschaffen werden, die im Bedarfsfall eine Kooperation zur Kinder- und Jugendpsychiatrie ermöglichen. Für die Arbeit mit dem Familiensystem gilt es, ein ausreichendes Zeitkontingent sowie eine klare Struktur und inhaltliche Ausrichtung zu etablieren. Implizit zeigt sich, dass zu allen am Hilfeprozess Beteiligten eine umfassende Vernetzung erstrebenswert wäre. Die Umsetzung dieser Aspekte sollte im Hinblick auf die Zielsetzung der Sicherung und Förderung kindlicher Entwicklung geschehen. Da die Perspektivklärung ein essenziell lebensverändernder Prozess für die Kinder ist, sollte es unumgänglich sein, diese ihrem Entwicklungsstand entsprechend partizipativ einzubeziehen.

\subsubsection{Limitationen}

Bei der vorliegenden Studie handelt es sich um eine explorative und nicht von Hypothesen geleitete Studie. Die Ergebnisse sind damit ein erster Hinweis zur Verbesserung der Prozessqualität in Perspektivklärung. Die Anzahl der ausgefüllten Fra- 
gebögen lassen keine Rückschlüsse über die Anzahl der teilgenommenen Gruppen zu. Selektionseffekte sind nicht auszuschließen, da sich die Gruppe der Fachkräfte, die den Online-Fragebogen vollständig ausgefüllt haben, systematisch von der Gruppe unterscheiden könnten, welche diesen nicht beantworteten. Allerdings begünstigt die gewählte Form der anonymen Befragung das Ermitteln der subjektiven Sichtweise, indem schambesetzte Themen eher geäußert werden (Wagner und Hering 2014). Die vorliegende Studie wurde fachkräftezentriert konzipiert. Nicht oder nur in eingeschränktem Umfang konnte ermittelt werden, inwieweit das jeweilige Perspektivklärungsverfahren aus Sicht der Kinder als erfolgreich eingestuft wird und welche Unterschiede dabei durch die divergierenden Vorgehensweisen zu begründen wären. Die explorativ gewonnenen Ergebnisse gilt es nun zu untermauern und zu erweitern.

Funding Open Access funding enabled and organized by Projekt DEAL.

Open Access Dieser Artikel wird unter der Creative Commons Namensnennung 4.0 International Lizenz veröffentlicht, welche die Nutzung, Vervielfältigung, Bearbeitung, Verbreitung und Wiedergabe in jeglichem Medium und Format erlaubt, sofern Sie den/die ursprünglichen Autor(en) und die Quelle ordnungsgemäß nennen, einen Link zur Creative Commons Lizenz beifügen und angeben, ob Änderungen vorgenommen wurden.

Die in diesem Artikel enthaltenen Bilder und sonstiges Drittmaterial unterliegen ebenfalls der genannten Creative Commons Lizenz, sofern sich aus der Abbildungslegende nichts anderes ergibt. Sofern das betreffende Material nicht unter der genannten Creative Commons Lizenz steht und die betreffende Handlung nicht nach gesetzlichen Vorschriften erlaubt ist, ist für die oben aufgeführten Weiterverwendungen des Materials die Einwilligung des jeweiligen Rechteinhabers einzuholen.

Weitere Details zur Lizenz entnehmen Sie bitte der Lizenzinformation auf http://creativecommons.org/ licenses/by/4.0/deed.de.

\section{Literatur}

Allroggen, M., Gerke, J., Rau, T., \& Fegert, J. (2016). Umgang mit sexueller Gewalt. Eine praktische Orientierungshilfe für pädagogische Fachkräfte in Einrichtungen für Kinder und Jugendliche. Ulm: Universitätsklinikum.

Baierl, M. (2014). Herausforderung Alltag. Praxishandbuch für die pädagogische Arbeit mit psychisch gestörten Jugendlichen. Göttingen: Vandenhoeck \& Ruprecht.

Beck, N. (2015). Jugendhilfebedarf nach (teil-)stationärer kinder- und jugendpsychiatrischer Behandlung. Eine deskriptive Analyse. Zeitschrift für Kinder- und Jugendpsychiatrie und Psychotherapie, 43, 443-453.

Bundschuh, C. (2010). Sexualisierte Gewalt gegen Kinder in Institutionen. Nationaler und internationaler Forschungsstand. Expertise im Rahmen des Projekts „Sexuelle Gewalt gegen Mädchen und Jungen in Institutionen“. München: Deutsches Jugendinstitut e. V.

Esser, G., \& Schmidt, M. (2017). Die Mannheimer Risikokinderstudie. Kindheit und Entwicklung, 26(4), 198-202.

Euser, S., Alink, L. R. A., Tharner, A., van Ijzendoorn, M. H., \& Bakermans-Kranenburg, M. J. (2013). The prevalence of child sexual abuse in out-of-home care: a comparison between abuse in residential and in foster care. Child Maltreatment, 18, 221-223.

Fegert, J., \& Petermann, F. (2014). Kinder- und Jugendpsychiatrie/Psychotherapie versus Kinder- und Jugendhilfe. Kindheit und Entwicklung, 23(3), 135-139.

Fischer, J., \& Geene, R. (2019). Gelingensbedingungen der Kooperation von Kinder- und Jugendhilfe und Gesundheitswesen: Handlungsansätze und Herausforderungen im Kontext kommunaler Präventionsketten. FGWStudie Vorbeugende Sozialpolitik, Bd. 19. Düsseldorf: Forschungsinstitut für 
gesellschaftliche Weiterentwicklung e. V. (FGW).. https://nbn-resolving.org/urn:nbn:de:0168-ssoar66316-2. Gesehen 25. Juni 2021.

Gadow, T., Peucker, C., Pluto, L., v. Santen, E., \& Seckinger, M. (2013). Wie geht's der Kinder- und Jugendhilfe? Empirische Befunde und Analysen. Weinheim Basel: Beltz Juventa.

Goldbeck, L., Besier, T., Schmid, M., \& Fegert, J. (2009). Aufforderung zur interdisziplinären pädagogischen und jugendpsychiatrischen Versorgung. Die Ulmer Studie zur psychischen Gesundheit von Kindern und Jugendlichen in Heimen. Kerbe. Forum für Sozialpsychiatrie, 2, 12-14.

Groen, G., \& Jörns-Presentati, A. (2014). An der Schnittstelle von stationärer Kinder- und Jugendhilfe und psychiatrisch-psychotherapeutischer Gesundheitsversorgung. Kindheit und Entwicklung, 23, 151-160.

Helming, E., Kindler, H., Langmeyer, A., Mayer, M., Mosser, P., Entleitner, C., Schutter, S., \& Wolff, M. (2011). Sexuelle Gewalt gegen Mädchen und Jungen in Institutionen. Abschlussbericht. München: Deutsches Jugendinstitut DJI.

[IKJ] Institut für Kinder- und Jugendhilfe Mainz. Caritas-Kinderheim gGmbH (2015). Damit es auf Dauer gelingt. Ambulante Familienarbeit und stationäre Therapeutische Übergangshilfe des Caritas-Kinder- und Jugendheimes Rheine im Vergleich. Mainz: Institut für Kinder- und Jugendhilfe Mainz.

Jozefiak, T., Kayed, N.S., Rimehaug, T., Wormdal, A. K., Brubakk, A. M., \& Wichstrøm, L. (2016). Prevalence and comorbidity of mental disorders among adolescents living in residential youth care. Eur Child Adolesc Psychiatry, 25(1), 33-47.

Jud, A., Rassenhofer, M., Witt, A., Münzer, A., \& Fegert, J. (2016). Häufigkeitsangaben zum sexuellen Missbrauch. Internationale Einordnung, Bewertung der Kenntnislage in Deutschland, Beschreibung des Entwicklungsbedarfs. Expertise. Arbeitsstab des Unabhängigen Beauftragten für Fragen des sexuellen Kindesmissbrauchs (Hrsg.). https://beauftragter-missbrauch.de/fileadmin/Content/pdf/ Pressemitteilungen/Expertise_H\%C3\%A4ufigkeitsangaben.pdf. Zugegriffen: 25. Juni 2021.

Kaufhold, G., Pothmann, J., \& Schilling, C. (2016). Junge Kinder in den Einrichtungen der stationären Erziehungshilfe. Auswertungen amtlicher Statistiken und Befragung der Jugendämter NRW zu jungen Kindern in stationären Einrichtungen. http://www.forschungsverbund.tu-dortmund.de/fileadmin/ Files/Hilfen_zur_Erziehung/290316_Junge_Kinder.pdf. Zugegriffen: 25. Juni 2021.

Knuth, N., \& Riehm, R. (2020). Elternarbeit und Elternpartizipation in der Inobhutnahme. In Fachgruppe Inobhutnahme (Hrsg.), Handbuch Inobhutnahme. Grundlagen - Praxis und Methoden - Spannungsfelder (S. 331-345). Frankfurt am Main: IgfH Eigenverlag.

Loch, U. (2018). Kinderschutz und Kooperation. Zur professionellen Gestaltung der Krisenintervention Inobhutnahme. Unsere Jugend, 2, 55-63.

[LWL] Landschaftsverband Westfalen-Lippe (2013). Hinweise zur Erteilung der Betriebserlaubnis von Einrichtungen und sonstigen Wohnformen nach § 45 SGB VIII. https://www.lwl-landesjugendamt. de/de/erzhilf/aufsicht-und-beratung-von-einrichtungen/materialien/\#anker-5800205. Zugegriffen: 25. Juni 2021.

Martín, E., González-García, C., Del Valle, J.F., \& Bravo, A. (2019). Detection of behavioral and emotional disorders in residential child care: Using a multi-informant approach. Children and Youth Services Review. https://www.sciencedirect.com/science/article/abs/pii/S0190740919307984. Zugegriffen: 25. Juni 2021.

Mayer, C. (2017). Die Bedeutung von Elternarbeit für die sozialpädagogische Praxis in stationären Einrichtungen der Kinder- und Jugendhilfe. Soziales_kapital, wissenschaftliches Journal österreichischer Fachhochschul-Studiengänge soziale Arbeit, 18, 75-90.

McKibbin, G. (2017). Preventing harmful sexual behaviour and child sexual exploitation for children \& young people living in residential care: a scoping review in the Australian context. Child and Youth Services Review, 82, 373-382.

Müller-Luzi, S., \& Schmid, M. (2017). Gelingensfaktoren und Stolpersteine in der Kooperation mit der Kinder- und Jugendpsychiatrie/-psychotherapie aus Sicht sozialpädagogischer Fachkräfte. Praxis der Kinderpsychologie und Kinderpsychiatrie, 66, 576-598.

Neumann-Witt, A. (2020). Vielfalt der Organisation Inobhutnahme gemäß $\$ 42$ SGB VIII. In Fachgruppe Inobhutnahme (Hrsg.), Handbuch Inobhutnahme. Grundlagen - Praxis und Methoden - Spannungsfelder (S. 39-57). Frankfurt am Main: IgfH Eigenverlag.

[Ofsted] Office for Standards in Education, Children's Services and Skills (2007). Children's messages on care. A report by the Children's Rights Director for England. London: [Ofsted] Office for Standards in Education, Children's Services and Skills.

[Ofsted] Office for Standards in Education, Children's Services and Skills (2010). Children's messages on care. A report by the children's rights director for England. Manchester: [Ofsted] Office for Standards in Education, Children's Services and Skills. 
[Ofsted] Office for Standards in Education, Children's Services and Skills (2011). The minister's discussion group with young people in residential care. A children's views report. London: [Ofsted] Office for Standards in Education, Children's Services and Skills.

Ortmann, K., \& Röh, D. (2017). Praxeologische Herausforderungen an die Sozialtherapie. Klinische Sozialarbeit. Zeitschrift für psychosoziale Praxis und Forschung. Themenschwerpunkt: Sozialtherapie, 13(1), 6-8.

Pauls, H. (2011). Klinische Sozialarbeit. Grundlagen und Methoden psychosozialer Behandlung (2. Aufl.). Weinheim, München: Juventa.

Pavel, J. R. (2016). Collaboration of parents and care professionals in cases of children placed in residential care homes. Parental Perspective. Athens Journal of Education, 3(1), 33-45.

Petermann, F., Besier, T., Büttner, P., Rücker, S., Schmid, M., \& Fegert, J. M. (2014). Vorläufige Schutzmaßnahmen für gefährdete Kinder und Jugendliche. Inobhutnahmen in Deutschland. Kindheit und Entwicklung. Zeitschrift für Klinische Kinderpsychologie, 23(2), 124-133.

Rücker, S., \& Büttner, P. (2017). Beteiligung von Kindern und Jugendlichen in der Inobhutnahme (\$42, SGB VIII): Welche Rolle spielen partizipative Prozesse im Kinderschutz?“. Blickpunkt Jugendhilfe, $5,10-14$.

Rücker, S., Büttner, P., Fegert, J.M., \& Petermann, F. (2015a). Partizipation traumatisierter Kinder und Jugendlicher bei vorläufigen Schutzmaßnahmen (Inobhutnahme, §42, SGB VIII). Zeitschrift für Kinder- und Jugendpsychiatrie und Psychotherapie, 43(5), 357-364.

Rücker, S., Büttner, P., Böge, I., Koglin, U., Fegert, M.J., \& Petermann, F. (2015b). Belastungen bei Kindern und Jugendlichen in der Inobhutnahme. Eine Analyse von Fallberichten. Nervenheilkunde, 34(01/02), 43-48.

Rudeck, R., Straus, F., \& Sierwald, W. (2008). Erfahrungen, Erkenntnisse und Ergebnisse. Baustein I: Repräsentative Befragung von Jugendlichen. In S. Hartig \& M. Wolff (Hrsg.), Forschungs- und Entwicklungsprojekt. Gelingende Beteiligung im Heimalltag aus Sicht von Jugendlichen. Projektzeitraum 8/2006-8/2008 (S. 56-63). SOS-Kinderdorf e. V.

Schleiffer, R. (2018). Fremdplatzierung und Bindungstheorie - Pflegekinder. In Stiftung zum Wohl des Pflegekindes (Hrsg.), Ein Pflegekind werden. Kindzentrierte Beiträge zur Inobhutnahme, Begutachtung, Perspektivklärung und Begleitung der Herkunftsfamilie (S. 135-168). Idstein: Schulz-Kirchner Verlag $\mathrm{GmbH}$.

Schmid, M. (2012). Psychisch belastete Heimkinder - eine besondere Herausforderung für die Schnittstelle zwischen Klinischer Sozialarbeit und Kinder- und Jugendpsychiatrie/-psychotherapie. In S. Gahleitner \& G. Hahn (Hrsg.), Klinische Sozialarbeit. Gefährdete Kindheit. Risiko, Resilienz und Hilfen. Beiträge zur psychosozialen Praxis und Forschung 3. (S. 113-121). Bonn: Psychiatrie Verlag.

Schuhrke, B. (2015). Die psychosexuelle Entwicklung des Kindes. Eine Betrachtung aus der Perspektive empirischer Forschung. Frühe Kindheit, 6(15), 50-55.

Schuhrke, B., \& Arnold, J. (2009). Kinder und Jugendliche mit problematischem sexuellen Verhalten in (teil-)stationären Hilfen zur Erziehung. Praxis der Kinderpsychologie und Kinderpsychiatrie, 58(3), $186-214$.

Sinclair, L., Vieira, M., \& Zufelt, V. (2019). Youth engagement and participation in a child and youth care context. Scottish Journal of Residential Child Care, 18(1), 29-47.

Statistisches Bundesamt (2018). Statistiken der Kinder- und Jugendhilfe. Vorläufige Schutzmaßnahmen. https://www.destatis.de/DE/Themen/Gesellschaft-Umwelt/Soziales/Kinderschutz/Publikationen/ Downloads-Kinderschutz/vorlaeufige-schutzmassnahmen-5225203187004.html. Zugegriffen: 25. Juni 2021 .

Timonen-Kallio, E. (2019). Interprofessional collaboration between residential child care and mental care practitioners: a cross-country study in six European countries. European Journal of Social Work, 22(6), 947-960.

Wagner, P., \& Hering, L. (2014). Online-Befragung. In N. Baur \& J. Blasius (Hrsg.), Handbuch Methoden der empirischen Sozialforschung (S. 661-673). Wiesbaden: Springer VS.

Walpot, M., Riis Hansen, G., Moentjes, G., \& Bernaards, C. (2017). Competencies for supporting a healthy sexual development of young people in care: interviews with a selection of professionals in Belgium, Denmark and The Netherlands. Hogeschool van Amsterdam: Amsterdams Kenniscentrum voor Maatschappelijke Innovatie.

[WHO und BZgA] WHO-Regionalbüro für Europa und BZgA (2011). Standards für die Sexualaufklärung in Europa. Rahmenkonzept für politische Entscheidungsträger, Bildungseinrichtungen, Gesundheitsbehörden, Expertinnen und Experten. Köln: Bundeszentrale für gesundheitliche Aufklärung. https:// www.bzga-whocc.de/fileadmin/user_upload/WHO_BZgA_Standards_deutsch.pdf. Zugegriffen: 25. Juni 2021. 
Zitelmann, M. (2010). Inobhutnahme und Kindesschutz. Ergebnisse einer bundesweiten Studie. Praxis und Forschung, Bd. 31. Frankfurt am Main: IgfH-Eigenverlag.

Zitelmann, M. (2013). Kindeswohlgefährdung und Inobhutnahme. Hinweise und Ergebnisse aus einer bundesweiten Studie. In G. Lewis, R. Riehm, A. Neumann-Witt, L. Bohnstengel, S. Köstler \& G. Hensen (Hrsg.), Inobhutnahme konkret (2. Aufl. S. 75-102). Frankfurt am Main: IgfH-Eigenverlag.

Sascha Dalügge erwarb während des Studiums der Sozialen Arbeit im Bachelor praktische Erfahrungen im Bereich der Perspektivklärungsgruppen und vertiefte diese während des klinisch-herapeutischen Masterstudiengangs auf Forschungsebene. Seit 2019 arbeitet er im LVR-Klinikum Düsseldorf im Krisen- und Aufnahmebereich für Jugendliche. Gegenwärtig absolviert er eine Weiterbildung zum Sexualpädagogen und nimmt einen Lehrauftrag an der Katholischen Hochschule NRW wahr.

Jenni Walther arbeitete nach ihrem Bachelor in Sozialer Arbeit in einer Perspektivklärungsgruppe. Während des anschließenden klinisch-therapeutischen Masterstudiengangs legte sie den Schwerpunkt auf die Erforschung der Perspektivklärung. Seit 2020 arbeitet sie in der Tagesklinik Walstedde im Kinder- und Jugendlichenbereich und nimmt einen Lehrauftrag an der Katholischen Hochschule NRW wahr.

Johanna Schratz studierte Sozialpädagogik und Soziale Arbeit im Bachelor an der Katholischen Hochschule in Münster. Im Anschluss daran war sie drei Jahre in einer Interdisziplinären Frühförder- und Beratungsstelle im Landkreis Leipzig tätig, bevor sie ihren Master mit dem Schwerpunkt Klinisch-therapeutische Soziale Arbeit an der Katholischen Hochschule in Aachen absolvierte. Seit 2020 arbeitet sie im Sachgebiet Ambulante Hilfen zur Erziehung des Jugendamtes der Stadt Wesseling und befindet sich derzeit in einer Weiterbildung zur Systemischen Einzel-, Paar- und Familientherapeutin.

Nicola Großheinrich studierte Psychologie an der Universität Bielefeld und promovierte anschließend in der Erwachsenenpsychiatrie der LMU München. Sie arbeitete in den Kinder- und Jugendpsychiatrien München, Aachen und Köln und erhielt 2016 den Ruf als Hochschulprofessorin an die Katholische Hochschule NRW für das Fachgebiet Psychische und Entwicklungsstörungen des Kindes- und Jugendalters. 